\title{
Performance Verification on UWB Antennas for Breast Cancer Detection
}

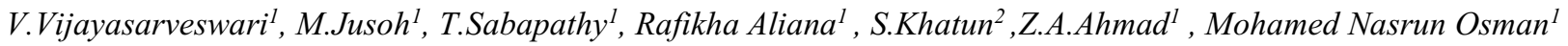 \\ ${ }^{1}$ Embedded Network and Advance Computing Research Cluster (ENAC), School of Computer and Communication Engineering, \\ Universiti Malaysia Perlis, Perlis. \\ ${ }^{2}$ Faculty of Electrical \& Electronic Engineering, Universiti Malaysia Pahang, Pekan, Pahang.
}

\begin{abstract}
Breast cancer is a common disease among women and death figure is continuing to increase. Early breast cancer detection is very important. Ultra wide-band (UWB) is the promising candidate for short communication applications. This paper presents the performance of different types of UWB antennas for breast cancer detection. Two types of antennas are used i.e: UWB pyramidal antenna and UWB horn antenna. These antennas are used to transmit and receive the UWB signal. The collected signals are fed into developed neural network module to measure the performance efficiency of each antenna. The average detection efficiency is $88.46 \%$ and $87.55 \%$ for UWB pyramidal antenna and UWB horn antenna respectively. These antennas can be used to detect breast cancer in the early stage and save precious lives.
\end{abstract}

\section{Introduction}

Breast cancer cases are increasing year by year. 100,000 cancer cases are reported every year in Malaysia. The five main cancers are breast cancer $(14.5 \%)$, internal cancer $(12.1 \%)$, lung cancer $(11.8 \%)$, cervical cancer $(5.7 \%)$ and throat cancer (5.4\%) [1]. Breast cancer is a leading case in Malaysia. However, $89 \%$ of women survived after breast cancer detected in early stage [2]. The available breast cancer detection methods are mammography, magnetic resonance imaging (MRI) and ultrasound.

Mammography is a technology that compresses the breast between two plates to get an X-ray image of the breast. Magnetic resonance imaging (MRI) scan is done by constructing images of the internal breast structure. It is using high magnetic field and radio energy wave. Ultrasound is using sound wave to image the breast structure [3-5]. Despite of the negative effect on human health, these types of methods are able to detect breast cancer but not in early stage [6-8]. If a cancer is detected, usually the patient has to undergo biopsy for further investigation. A wrong detection can make patient undergo some painful diagnose process. This traditional equipment are only available in some of the clinics and hospitals because they are very expensive $[9,10]$. Moreover, this equipment should be handled by an expert operator. So, the patient needs to go to the specific hospital for their check-up. These types of screening systems are not advisable to use regularly due to their side effects. Besides, the detected cancer cannot be determined whether it is benign or malignant by the available equipment except biopsy $[5,8]$. Determining of benign or malignant is important for further treatment decision.

To overcome the above shortcomings, researchers have been proposed new breast cancer detection system using microwave based UWB imaging technology. Radar based imaging method is widely used for breast cancer detection compared to microwave tomography $[9,11,12]$. There are a lot of ways to process the received signal. Most of the researchers used VNA to view the received signal. The antenna is attached to the VNA to obtain output and the image of the breast $[13,14]$. In other hand, researcher has used real time oscilloscope to simulate the received signal from pulse generator [15]. Some researchers have proposed to use artificial intelligence (AI) to analyse the received UWB signal for early tumor detection $[4,12,16]$. While antenna will transmit and receive the signal. This signal contains the signature of the cancer. Different type of antennas included radar antenna [17], Vivaldia antenna [18,19], horn antenna [20,21], slot on-body antenna [22] and wideband half oval patch antenna, stacked patch antenna and wide-slot antenna [23] have been designed and used to detect breast cancer. Here, the performance of two types of antennas (wearable UWB pyramidal antenna and UWB horn antenna) are analysed to detect the breast cancer.

\footnotetext{
* Corresponding author: M.Jusoh (muzammil@unimap.edu.my)
} 


\section{Methods and Material}

Figure 1 shows the summary of the methodology taken throughout the experiment. Firstly the forward scattered UWB signals are collected using the two types of antennas (UWB pyramidal and UWB horn antenna). Then, the collected UWB signals are converted into digital form (1632 data points). The data samples are combined together and fed into the developed k-fold based feed forward backpropagation neural network (KFFBPNN) module. KFFBPNN module is trained, validated and tested until it is optimized. The developed KFFBPNN module is used to test using untrained data samples.

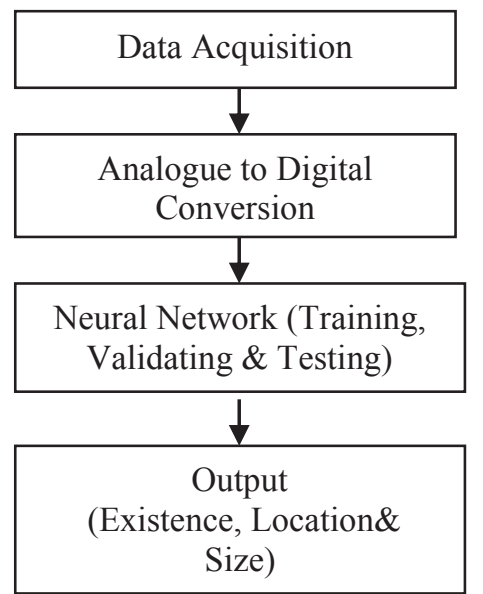

Figure 1: Summary of Methodology

\subsection{Antenna Design}

Two types of antennas are used here: UWB pyramidal antenna and UWB horn antenna. Both antennas are designed in Computer System Technology (CST) Microwave Studio in frequency range of $3.1 \mathrm{GHz}-$ $10 \mathrm{GHz}$. Both UWB pyramidal antenna and UWB horn antenna is fabricated using textile (Zelt textile for patch and Felt textile for substrate) as shown in Figure 2.

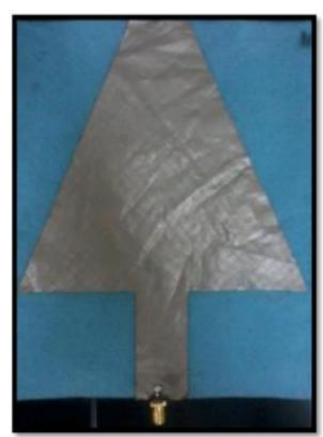

(a) Pyramidal Antenna

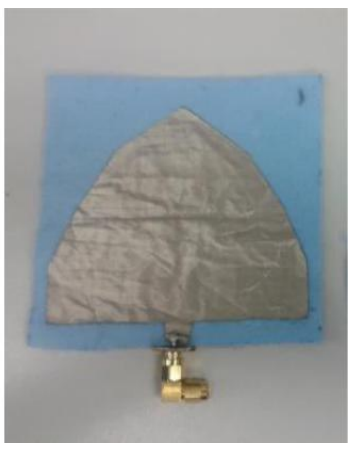

(b) Horn Antenna
Figure 2: UWB Antenna

\subsection{Data Acquisition}

Heterogeneous breast phantom and tumor are developed with existing chemical materials as shown in Figure 3 [25]. The phantom consists of fat, gland and skin. Tumor is developed with different sizes $(3 \mathrm{~mm}$ and $5 \mathrm{~mm})$. The materials are included p-toluic acid, n-propanol, deionized water, 200 bloom gelatine, formaldehyde, safflower oil and ultra-ivory detergent. The amount of each material is different for fat, gland, skin and tumor respectively.

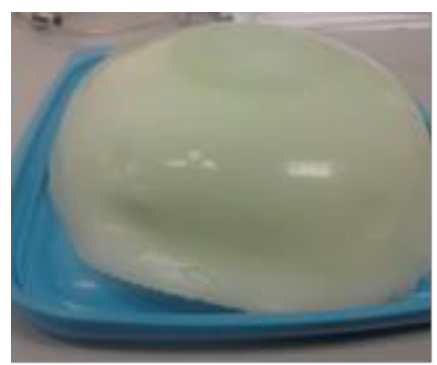

Figure 3: Heterogeneous Breast Phantom

The breast phantom is placed in the middle of a pair of antenna as shown in Figure 4. Antenna will transmit UWB signal and another antenna will receive the UWB signal. This UWB signal contains the information of tumor's location and size. The received UWB signals are saved in PC for further investigation. UWB signals are initially in analogue and converted to digital through Analogue to Digital Conversion.

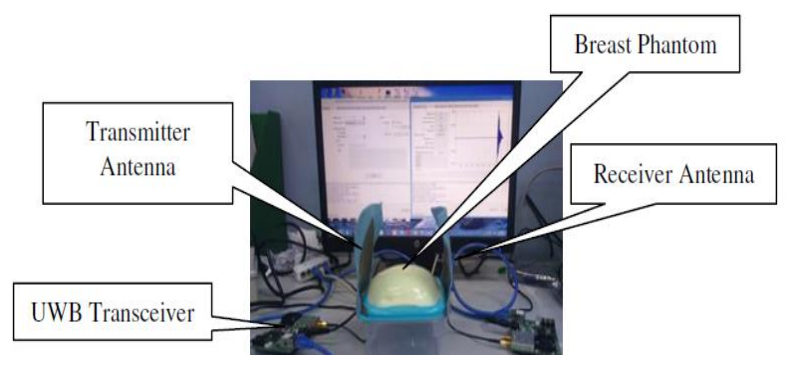

Figure 4: Experiment Set-up

\subsection{Neural Network}

The 82 data samples are collected for UWB pyramidal antenna (41 data samples) and UWB horn antenna (41 data samples). 41 data samples were divided into two groups. First group consisted of 30 data samples for training, validating and testing while second group consisted of 11 data samples for real time testing. Each data sample consisted 1632 data points. Four data points are left after extracted the important points from 1632 data points. Four data points included mean, median, maximum number and minimum number.

K-fold based feed forward backpropagation neural network (c) module is developed in Matlab software. Hidden neuron, hidden layer and training function are 
the most important parameter during developing the KFFBPNN module. These parameters are changed depending on input data and target data. First group data samples (30 data samples) are fed into the KFFBPNN module to train, validate and test the network. The network is trained continuously until it is optimized but network should not memorize the output. The optimized network parameters are shown in Table 1.

Table 1: Network Parameters

\begin{tabular}{|l|l|}
\hline Neural Network Parameters & \\
\hline Number of nodes in input layer & 4 \\
\hline Number of nodes in first hidden layer & 20 \\
\hline Number of nodes in second hidden layer & 1 \\
\hline Number of nodes in output layer & 4 \\
\hline Training function & Trainlm \\
\hline Learning rate & 0.009 \\
\hline Momentum constant & 0.6 \\
\hline Maximum number of epochs & 1000 \\
\hline Minimum performance gradient & $1 \mathrm{e}-25$ \\
\hline
\end{tabular}

Table 2: Detection Performance Efficiency for UWB Pyramidal Antenna

\begin{tabular}{|c|c|c|r|r|r|r|r|r|r|r|r|}
\hline \multicolumn{4}{|c|}{ Actual Target (mm) } & \multicolumn{4}{c|}{ NN output (mm) } & \multicolumn{3}{|c|}{ Detection Performance Efficiency } \\
\hline $\mathrm{x}$ & $\mathrm{y}$ & $\mathrm{z}$ & size & $\mathrm{x}$ & $\mathrm{y}$ & $\mathrm{z}$ & \multicolumn{1}{c|}{ size } & $\mathrm{x}$ & $\mathrm{y}$ & $\mathrm{z}$ & \multicolumn{1}{c|}{ size } \\
\hline-1 & -1 & -1 & -1 & -1.16 & -0.86 & -0.33 & -1.00 & 100 & 100 & 100 & 100 \\
\hline 32.5 & 32.5 & 40 & 3 & 36.04 & 16.35 & 35.79 & 13.62 & 90.28 & 50.15 & 89.50 & 45.33 \\
\hline 32.5 & 32.5 & 40 & 5 & 31.69 & 19.62 & 67.02 & 50.00 & 97.54 & 60.30 & 32.50 & 100 \\
\hline 62.5 & 32.5 & 30 & 3 & 59.76 & 40.43 & 59.59 & 29.98 & 95.52 & 80.45 & 50.34 & 99.67 \\
\hline 32.5 & 62.5 & 30 & 3 & 48.78 & 62.49 & 58.65 & 29.99 & 66.60 & 99.84 & 51.19 & 99.67 \\
\hline 32.5 & 1.0 & 30 & 3 & 31.00 & 1.96 & 28.51 & 29.98 & 95.38 & 96.92 & 95.00 & 99.67 \\
\hline 62.5 & 32.5 & 30 & 5 & 67.18 & 32.31 & 28.73 & 50.00 & 93.14 & 99.38 & 95.67 & 100 \\
\hline 1.0 & 32.5 & 30 & 3 & 49.85 & 29.01 & 49.59 & 23.92 & 48.50 & 89.23 & 60.61 & 79.67 \\
\hline 32.5 & 1.0 & 30 & 5 & 42.11 & 0.88 & 30.27 & 50.01 & 77.20 & 90.00 & 99.34 & 99.80 \\
\hline 32.5 & 62.5 & 30 & 5 & 30.20 & 62.57 & 29.20 & 44.83 & 92.92 & 99.84 & 97.33 & 89.60 \\
\hline 1.0 & 32.5 & 30 & 5 & 2.69 & 33.59 & 25.94 & 53.30 & 80.00 & 96.73 & 86.33 & 93.98 \\
\hline
\end{tabular}

Table 3: Detection Performance Efficiency for UWB Horn Antenna

\begin{tabular}{|c|c|c|c|c|l|l|l|l|l|c|c|}
\hline \multicolumn{4}{|c|}{ Actual Target (mm) } & \multicolumn{4}{c|}{ NN output (mm) } & \multicolumn{4}{c|}{$\begin{array}{c}\text { Detection Performance } \\
\text { Efficiency (\%) }\end{array}$} \\
\hline $\mathrm{x}$ & $\mathrm{y}$ & $\mathrm{z}$ & size & $\mathrm{x}$ & $\mathrm{y}$ & $\mathrm{z}$ & size & $\mathrm{x}$ & $\mathrm{y}$ & $\mathrm{z}$ & size \\
\hline-1 & -1 & -1 & -1 & -0.53 & -0.85 & -1.24 & -0.66 & 100 & 100 & 100 & 100 \\
\hline 32.5 & 32.5 & 40 & 3 & 45.17 & 27.19 & 8.23 & 3.62 & 61.02 & 83.65 & 20.59 & 79.49 \\
\hline 32.5 & 32.5 & 40 & 5 & 35.16 & 34.76 & 39.99 & 4.66 & 91.81 & 93.05 & 99.99 & 93.19 \\
\hline 62.5 & 32.5 & 30 & 3 & 54.74 & 34.21 & 29.98 & 2.99 & 87.52 & 94.75 & 99.97 & 99.97 \\
\hline 32.5 & 62.5 & 30 & 3 & 36.17 & 67.84 & 29.99 & 1.83 & 88.72 & 91.46 & 99.99 & 61.01 \\
\hline 32.5 & 1.0 & 30 & 3 & 42.88 & 0.52 & 29.82 & 4.12 & 68.05 & 52.10 & 99.42 & 62.65 \\
\hline 62.5 & 32.5 & 30 & 5 & 67.17 & 24.19 & 30.00 & 5.09 & 92.53 & 74.42 & 100 & 98.22 \\
\hline 1.0 & 32.5 & 30 & 3 & 5.07 & 44.12 & 8.34 & 2.99 & 49.70 & 64.23 & 27.84 & 99.68 \\
\hline 32.5 & 1.0 & 30 & 5 & 69.86 & 43.64 & 29.60 & 4.53 & 46.56 & 22.94 & 9.87 & 90.51 \\
\hline 32.5 & 62.5 & 30 & 5 & 28.06 & 59.86 & 29.98 & 5.01 & 86.35 & 95.78 & 99.95 & 99.82 \\
\hline 1.0 & 32.5 & 30 & 5 & 4.19 & 64.87 & 30.16 & 5.08 & 94.88 & 50.15 & 99.45 & 98.37 \\
\hline
\end{tabular}

Table 4 showed the average detection efficiency for UWB pyramidal antenna and UWB horn antenna. UWB pyramidal antenna $(88.46 \%)$ performed better than UWB horn antenna $(87.55 \%)$. The antenna is fabricated using the same materials but in different size. UWB pyramidal

\section{Results and Discussion}

After the training session, the developed KFFBPNN module is tested using untrained 11 data samples from second group. This process is essential to determine the efficiency of developed module. Table 2 and Table 3 showed the detection accuracy for each output data and detection performance efficiency for UWB pyramidal antenna and UWB horn antenna respectively. Here to mention, negative value indicated the absence of the tumor while positive value indicated the presence of the value.

antenna is fabricated in larger size compared to the UWB horn antenna. UWB pyramidal antenna is able to close the phantom completely and able to transmit and receive all the UWB signals without loss any signals. 
Table 4: Average Detection Efficiency

\begin{tabular}{|c|c|c|c|c|}
\hline \multicolumn{2}{|c|}{ Antenna Type } & $\begin{array}{c}\text { UWB } \\
\text { Pyramidal } \\
\text { Antenna }\end{array}$ & $\begin{array}{c}\text { UWB } \\
\text { Horn } \\
\text { Antenna }\end{array}$ \\
\hline $\begin{array}{c}\text { Performance } \\
\text { Efficiency } \\
(\%)\end{array}$ \\
\cline { 2 - 5 } & \multicolumn{2}{|c|}{ Existence } & 100 & 100 \\
\cline { 3 - 5 } & Location & $\mathbf{x}$ & 85.19 & 79.43 \\
\cline { 2 - 5 } & $\mathbf{y}$ & 87.53 & 81.09 \\
\cline { 2 - 5 } & $\mathbf{z}$ & 77.98 & $\begin{array}{c}96 . \\
17\end{array}$ \\
\cline { 2 - 5 } & \multicolumn{2}{|c|}{ Size } & 91.58 & 93.50 \\
\hline \multicolumn{2}{|c|}{ Average Detection Efficiency (\%) } & 88.46 & 87.55 \\
\hline
\end{tabular}

\section{Conclusions}

Two types of antennas are used to analyse its performance for breast cancer detection. The designed antennas are fabricated using textile materials so that it can be wear by the patient during the screening process. $100 \%$ detection efficiency is achieved in terms of existence of the tumor. The developed system and wearable antenna able to detect breast cancer in the early stage without any difficulties. This will help to save precious lives.

\section{Acknowledgement}

This work is supported by Ministry of Higher Education, Malaysia, Grant FRGS - 9003-00418

\section{References}

1. The Star Online. Retrieved from http://www.thestar.com.my/news/nation/2016/04/03 /about-100000-malaysians-suffer-from-cancer-eachyear/ (Date accessed: 6 April 2017)

2. C.H. Yip, P.N. Bhoo, S.H. Teo, Med J Malaysia, 69, 8 (2014).

3. T. Henriksson, M. KlemM, D. Gibbins, J. Leendertz, T. Horseman, A.W. Preece, R. Benjamin, I.J. Craddock. Antennas and Propagation Conference (LAPC), 1 (2011).

4. K.J. Reza, S. Khatun, M. Jamlos, M. Fakir, M.N. Morshed, Journal of Medical Imaging and Health Informatics, 5, 1246 (2015).

5. M. Løberg, M.L. Lousdal, M. Bretthauer, M. Kalager, Breast Cancer Research, 17, 63 (2015).

6. M. Sajjadieh, F. Foroozan, A. Asif, 13th IEEE International Multitopic Conference, 1 (2009).

7. H. Sylvia, Heywang-Köbrunner, A. Hacker, S. Sedlacek, Breast Cancer (Basel), 6, 199 (2011).

8. A. Jalalian, S. Mashohor, R. Mahmud, B. Karasfi, M.I. Saripan, A.R. Ramli, Excli Journal, 16, 113 (2017).

9. V.Vijayasarveswari, S. Khatun, M.Jusoh, M.M. Fakir, Journal of Scientific Research and Development, 3, 59 (2016).
10. S.D. Placido, C.D. Angelis, M. Giuliano, C. Pizzi, R. Ruocco, V. Perrone, D. Bruzzese, G. Tommasielli, M.D. Laurentiis, S. Cammarota, G. Arpino, British Journal of Cancer, 116, 821 (2017).

11. K.J. Reza, S. Khatun, M.F. Jamlos, M.M. Fakir, Ikram-e-Khuda, Z. Ishwar, International Journal of Engineering and Technology, 5 (2013).

12. S.A. Alshehri, S. Khatun, Progress In Electromagnetics Research C, 7, 79 (2009).

13. S.H.M. Salleh, M.A. Othman, N. Ali, H.A. Sulaiman, M.H. Misran, M.Z.A.A. Aziz, ARPN Journal of Engineering and Applied Sciences, 10, 723 (2015).

14. M. Klemm, J.A. Leendertz, D. Gibbins, I.J. Craddock, A. Preece, R. Benjamin, Antennas and Wireless Propagation Letters, IEEE, 8, 1349 (2009).

15. J.C.Y. Lai, C.B. Soh, E. Gunawan, K.S. Low, Progress In Electromagnetics Research M, 16, 19 (2011).

16. S.A. Alshehri, S. Khatun, A.B. Jantan, R.S.A.R. Abdullah, R. Mahmood, Z. Awang, Progress In Electromagnetics Research, 111, 447 (2011).

17. A.W. Preece, I. Craddock, M. Shere, L. Jones, H.L. Winton, Journal of Medical Imaging, 3, (2016).

18. Y. Medina, M. Augusto, A.V. Paz, 2016 IEEE Andescon, 1(2016).

19. C.J. Shannon, E.C. Fear, M. Okoniewski, Electronics Letters, 41, 388 (2005).

20. R.K. Amineh, A. Trehan, N.K. Nikolova, Progress In Electromagnetics Research B, 13, 59 (2009).

21. K. L. Narayanan, V. Ayyempillai, International Journal Of Innovative Research In Science, Engineering And Technology, 2, 508 (2013).

22. X. Xia, S. Hang, W.Z. Jie, W. Liang, Chin. Phys. B, 23, 1 (2014).

23. A. Sam, A.A. Jone.A, International Journal Of Computer Trends \& Technology, 1, 281 (2013).

24. E. Porter, J. Fakhoury, R. Oprisor, M. Coates, M. Popovic, Antennas and Propagation (EuCAP), 1 (2010). 